\title{
Failure to thrive: diagnostic yield of hospitalisation
}

\author{
DONALD M BERWICK, JANICE C LEVY, AND RUTH KLEINERMAN
}

Center for the Analysis of Health Practices, Harvard School of Public Health, and Department of Medicine, The Children's Hospital Medical Center, Boston, Massachusetts, USA

SUMmARY Review of hospital records of 122 infants, aged between 1 and 25 months, admitted to a teaching hospital with the diagnosis of failure to thrive but without an underlying disease apparent at admission, showed that about one-third of them still had no diagnosis after evaluation. Thirty-two per cent were thought to have a social or environmental explanation for poor growth, and $31 \%$ were given a specific organic or physiological diagnosis. Of the last group, 2 out of 3 were diagnosed as having either gastro-oesophageal reflux or non-specific chronic diarrhoea. Vomiting was often associated with organic or structural disease. On average about 40 laboratory tests and $x$-ray films were performed per infant, but only $0.8 \%$ of all tests showed an abnormality which contributed to the diagnosis of the cause of failure to thrive. Our results stress the importance of social and environmental factors as basic causes of failure to thrive, and suggest that admission to hospital and laboratory testing is unlikely to lead to a specific organic diagnosis in a child whose failure to thrive is unexplained after careful history taking and a physical examination.

One of the most vexing problems for clinicians caring for young children is the search for aetiologies in the management of small children who 'fail to thrive', children whose weight lies consistently below the 3rd centile for age, or whose growth is rapidly crossing centiles downwards. Such children account for between 1 and $5 \%$ of all admissions to referral children's hospitals, ${ }^{12}$ and many of these admissions are initiated to search for occult diseases underlying the growth failure.

The differential diagnosis of the condition is long, almost conterminous with the list of serious chronic diseases of childhood, and a diagnostic search which attempts to 'rule out' all possible organic causes of failure to thrive (FTT) is necessarily extensive. Apart from organic disease, the important association between adverse environmental factors and impaired growth and development is well known. ${ }^{34}$ Series report very different proportions of nonorganic aetiological classifications, some reporting a high percentage of FTT children with underlying organic problems, ${ }^{125-7}$ but others reporting a far lower one. ${ }^{89}$ Such differences seem mainly owing to variations in criteria.

Few of the series have dealt directly with the central problem of strategy for the clinician managing a small child with FTT; namely, what is the appropriate course of action if the patient has neither organic disease nor abuse or neglect as an obvious cause of the growth disturbance. In particular, how can ad- mission to hospital contribute to the resolution of the diagnostic dilemma? This study was undertaken to assess the diagnostic yield of children in the infant-toddler age group who are admitted to hospital to investigate the cause of FTT of obscure origin. In addition, we sought to determine whether any characteristics of the child definable at admission, any characteristics of growth in the hospital, or any laboratory results were strongly correlated with the discharge diagnosis.

\section{Methods}

We abstracted detailed information from the case records of all infants aged between 1 and 25 months for whom the diagnosis of FTT had been noted at admission to or on discharge from a paediatric teaching hospital during a 2-year period. Children were excluded from the study if an obvious cause of FTT was explicitly identified by history or physical examination at the time of admission, or if the infant was in a medically critical condition at the time of admission. One hundred and twenty-two cases met the criteria for inclusion.

Discharge diagnoses, as recorded by the house officer responsible for each case, were grouped into the following 4 categories.

Group 1 FTT unexplained. In this group no final cause, either organic or environmental, was found for the growth failure. 
Group 2 FTT environmental. This group comprised children whose FTT was directly attributed to an adverse social or physical environment.

Group 3 FTT specific diagnosis. This group comprised children whose FTT was attributed to a named physical dysfunction, whether functional or anatomic. For those with specific diagnoses, two subgroups were defined. Subgroup A comprised children with apparent disorders of gastrointestinal function, in whom growth failure seemed secondary to the chronic loss of nutrient through vomiting and diarrhoea, but who did not have a specific structural abnormality identified during their time in hospital. For these children, the discharge diagnoses often amounted to the re-naming of a symptom noted on admission-for example, FTT secondary to gastrooesophageal reflux, or FTT secondary to chronic diarrhoea. Subgroup B comprised children who had specific evidence of a known disease entity discovered during their duration in hospital. Their discharge diagnosis was the name of that disease.

Group 4 not FTT. A few children were admitted for the diagnosis of FTT but were finally judged to have normal growth.

In further analyses, these diagnostic groups serve as the major divisions of the cases in our study. These causal assignments are limited by the fact that they were subjective, but we believe that the diagnostic impression at discharge, although imperfect, is the best measure available of the diagnostic outcome of admission to hospital as it affects subsequent medical management.

\section{Results}

The distribution of discharge diagnoses in our series is shown in Table 1 . Overall, $34 \%$ had no diagnosis at the time of discharge, $32 \%$ had FTT ascribed to an environmental aetiology, and $31 \%$ were given a specific diagnosis. Of this last group, two-thirds had a functional gastrointestinal disorder (group 3A) and one-third had a specific structural disease (group 3B, Table 2). In the 38 children whose FTT was attributed to an organic process, the gastrointestinal tract was the site of that process in 31 $(82 \%)$. The distribution of diagnoses among the children with the most profound FTT was not statistically different from that for the remainder of the study group.

Admission characteristics. Three of the features noted on the admission histories were associated with ultimate diagnosis: Medicaid eligibility, growth pattern before admission, and history of vomiting. Birthweight, prematurity, and age at admission were
Table 1 Discharge diagnoses in 122 infants

\begin{tabular}{|c|c|c|}
\hline \multirow[b]{2}{*}{ Discharge diagnostic category } & \multicolumn{2}{|c|}{ Total children } \\
\hline & No & $\%$ \\
\hline $\begin{array}{l}1 \text { Unexplained } \\
\text { Unknown (33) } \\
\text { Constitutional (8) }\end{array}$ & 41 & 34 \\
\hline $\begin{array}{l}2 \text { Social-environmental } \\
\text { Social deprivation (22) } \\
\text { Malnutrition (17) }\end{array}$ & 39 & 32 \\
\hline $\begin{array}{l}\text { 3A Functional gastrointestinal } \\
\text { Oesophageal refiux (16) } \\
\text { Chronic diarrhoea (10) }\end{array}$ & 26 & 21 \\
\hline $\begin{array}{l}\text { 3B Specific structural } \\
4 \text { Not failure to thrive }\end{array}$ & 12 & 10 \\
\hline
\end{tabular}

Table 2 Specific structural causes of failure to thrive

\begin{tabular}{|c|c|}
\hline Discharge diagnosis & No of children \\
\hline $\begin{array}{l}\text { Partial intestinal obstruction } \\
\text { Pyloric stenosis (2) } \\
\text { Malrotation (1) }\end{array}$ & 3 \\
\hline Urinary tract infection & 3 \\
\hline Tuberculosis & 1 \\
\hline $\begin{array}{l}\text { Neurological } \\
\text { Leigh's disease (1) } \\
\text { Cerebral palsy (1) }\end{array}$ & 2 \\
\hline Coeliac disease & 2 \\
\hline Hypercalcaemia & 1 \\
\hline
\end{tabular}

not statistically associated with discharge diagnosis.

Medicaid-eligible families are those who, because of poverty or certain forms of disability, qualify for governmental payment for personal health care. In this study, Medicaid eligibility is used as an indicator of low socioeconomic status. Although Medicaid was the payment source for $29 \%$ of cases, Medicaideligible children accounted for $43 \%$ of those with the discharge diagnosis of socioenvironmental FTT $(\mathrm{P}<0.02)$.

Composite pre-hospital growth data on each diagnostic group were assembled and superimposed on the Boston anthropometric chart. The composite growth curve of the group with FTT unexplained at discharge (group 1) showed that these infants were following normal growth channels, although displaced downwards from the 3rd centile. In effect, $\frac{D}{O}$ they were growing at a normal rate, but were small, similar to children with genetic short stature. $\tilde{o}^{\circ}$ Children in group 2 with presumed environmental deprivation were characterised by an aggregated 0 growth curve further below the 3 rd centile than that $\mathrm{\omega}$ of group 1 , and deviating progressively with age from the normal channels. Children in group $3 \mathrm{~A}$, those with chronic diarrhoea or gastro-oesophageal reflux, had the most strikingly deviant growth curves in aggregate. Babies with the ultimate diagnoses sug- $\frac{T}{\circ}$ gesting oesophageal reflux seemed abnormal almost $\frac{0}{\mathbb{D}}$ from birth, with curves of nearly flat slope, deviating $\stackrel{\odot}{\odot}$ progressively from normal channels beginning very $\stackrel{\Phi}{\varrho}$ early in their lives. The group with chronic diarrhoea, 
on the other hand, appeared to grow well during the first 6 months of life, and then to deviate suddenly and markedly from normal with nearly 'horizontal' growth records from age 6 months onwards. These various growth patterns are matters of impression based on aggregated group data, but suggest that children with different causes of growth disturbances may have different longitudinal patterns of growth. Whether such different patterns reflect differences in the time of onset of the underlying condition is a question not settled by the data available to us.

A history of recurrent vomiting was unevenly distributed among diagnostic groups. Of the 19 children admitted with the chief complaint of FTT and vomiting, $60 \%$ were in group 3 at discharge compared with $26 \%$ of non-vomiters $(P<0.01)$, and all specific diagnoses among vomiters affected the gastrointestinal tract.

Hospital course. Some investigators have suggested that one may distinguish environmental from organic FTT by observing growth in a therapeutic environment-that those with environmental causes will gain, and those with organic disease will not. To test that observation, we recorded in-hospital growth for each of our diagnostic groups. On this variable, individual performance was erratic. Among children with socioenvironmental diagnoses, more children gained than lost weight during their time in hospital, but there were gainers and losers in each diagnostic group, and the hospital course was not generally a reliable indicator of the discharge diagnosis.
Laboratory investigations. Altogether 4827 tests of 126 different types were performed on 122 children in our series, or 39.6 tests per admission. The average admission lasted nine days. Tests accounted for $24 \%$ of the total costs of admission to hospital.

Analyses of the yield of these laboratory investigations focused on the information content of positive or abnormal results. No attempt was made to assess the undeniable value of negative or normal tests used to 'rule out' worrying diagnostic possibilities.

No single test discriminated non-specifically but reliably between organic and non-organic diagnostic groups. Although many abnormalities were noted, most tests did not contribute to diagnostic classification. Liver function test abnormalities for example, which occurred at least once in more than $75 \%$ of our sample, were no more common in one diagnostic group than in another. Other tests commonly found to be abnormal but not specific were bone age, microscopical stool fat and fibre, and endocrine tests (including measures of thyroid function and glucose metabolism).

Abnormal tests did assist diagnosis in a few cases. Table 3 summarises all instances in which a specific laboratory test abnormality contributed to a specific disease diagnosis. If either of two physician reviewers (DMB or $\mathrm{JCL}$ ) believed that an abnormality contributed to an understanding of a child's disease, the result was included in Table 3, even if redundant with other information.

In $19(16 \%)$ of the 122 cases a test abnormality

Table 3 Test abnormalities useful in diagnosing the cause of FTT

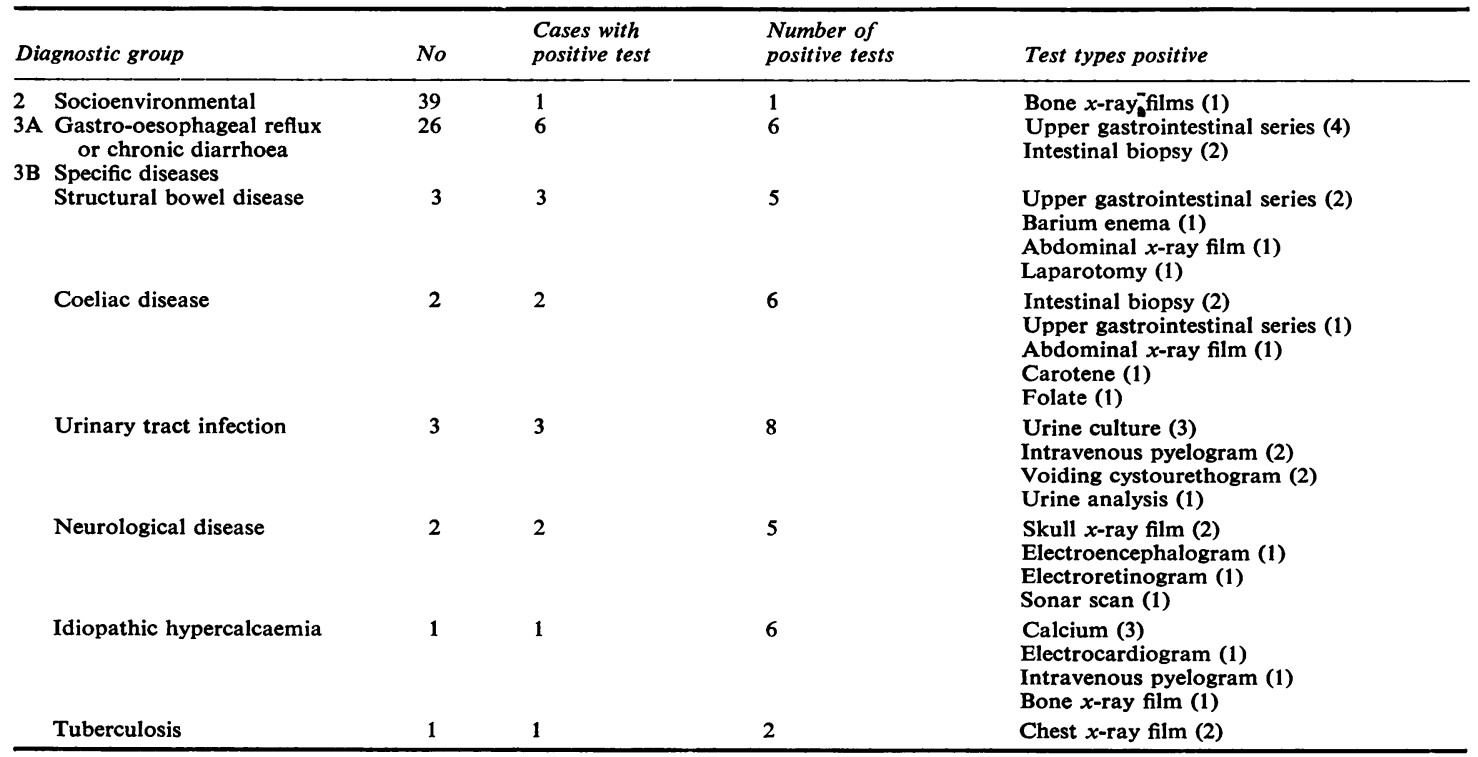


was of positive use in elucidating the cause of FTT. In these 19 cases, a total of 39 tests, or $0.8 \%$ of all tests done, made a positive contribution by these criteria. Studies of the gastrointestinal tract predominated in this accounting, with upper gastrointestinal series, intestinal biopsy, and abdominal $x$-ray films accounting for $13(33 \%)$ of the 39 tests.

Since some laboratory tests were presumably obtained to assist in the management of acute intercurrent conditions-such as fever or diarrhoeacases were individually reviewed to assess the usefulness of abnormal results for any purpose other than diagnosis of FTT. One hundred and seventyfour tests, or $3.6 \%$ of all tests done, were of some value in detecting or managing abnormalities other than FTT. Haematocrits in the diagnosis of anaemia were the most commonly useful tests and accounted for $60 \%$ of all useful investigations. Overall, excluding haematocrits, a total of 112 abnormal tests, or $2.5 \%$ of all non-haematocrit tests done, were of assistance in either diagnosing FTT or managing a concurrent condition.

\section{Discussion}

Our review of one hospital's recent experience in the inpatient evaluation of FTT shows a predominance of non-organic aetiologies underlying the growth failure among children who had no specific disease evident at the time of admission. Barely 1 child in 3 received a physical diagnosis after hospital evaluation, and two-thirds of those who were so labelled were thought to have functional gastrointestinal disturbances (oesophageal reflux or non-specific chronic diarrhoea), which may have a strong environmental component. In the series as a whole, only one child in 10 was found to have a specific structural disease other than gastro-oesophageal reflux or diarrhoea. This pattern was not significantly different even among children with the most profound growth failure.

Gastro-oesophageal reflux was the most common single specific diagnosis in our sample. Predictably, a history of vomiting was strongly associated with this diagnosis, as well as with structural bowel anomalies, and our review suggests that vomiting should serve as a target to guide initial diagnostic study.

The association noted between Medicaid eligibility and the diagnosis of socioenvironmental deficiences is easy to misinterpret. Although poverty has been linked in other series with non-organic FTT, the finding may simply reflect a tendency to label the poor as neglectful or inept parents when no other cause for FTT is discovered. Pollitt's ${ }^{10}$ controlled series of children with FTT did find more poverty among cases than among comparison families, but the association was not striking. Recent research suggests that non-organic FTT is more fruitfully viewed as a dynamic interactional problem in the mother-child dyad instead of as simply an abnormality of the child or an inadequacy of the parent or environment. Such interaction also helps to explain why some children with physical disease or functional intestinal problems fail to thrive while others with similar physical problems cope well and grow, or why one child in a family fails while siblings are not affected.

Our series suggests that the hospital laboratory holds very limited promise in elucidating FTT. In these cases, fewer than $1 \%$ of the tests showed abnormalities which helped to explain the cause of FTT, affecting diagnoses in only $16 \%$ of the children (findings which conform closely to those of Sills). ${ }^{9}$ Laboratory and radiological study of the gastrointestinal tract was more frequently productive of diagnostic information than other types of study. We did not estimate the undeniable value of some tests in ruling out diagnoses of concern.

Our observations should be treated with caution. We have no assurance that our sample is representative of infants in hospital elsewhere, and our assignment of cases to diagnostic categories based on discharge notes dictated by a large number of houseofficers cannot be subjected to adequate tests of inter-observer reliability nor are they of predictive validity. Prospective studies are needed to validate these diagnostic classifications by more objective criteria and to supply information on the variations in the natural history and outcome of different diagnostic subgroups.

In terms of achieving diagnostic certainty, our findings challenge the usefulness of current inpatient strategies. There may have been benefits not assessed by our methods-such as reassurance to parents, nutrition education or, in a few cases, the initiation of legal steps concerning the child's care-but, on the whole, the volume of laboratory investigation and the apparent aggressiveness with which organic explanations were pursued without much success suggests an imbalance in the attention of the $\widetilde{N}$ responsible physicians. If the reason for FTT is N unknown, and if careful and thorough initial history taking and examination fail to offer clues about $\omega$ underlying disease, the pursuit of organic diagnosis should be tempered by increased attention to the nurturant interactions of parents and child. That $\stackrel{\infty}{\Phi}$ attention can be diverted and those interactions can be distorted by a hospital that places too much emphasis on the search for unitary causes of a highly complex problem.

This study was supported in part by grants fromg 
the Spencer Foundation through the Harvard Graduate School of Education, and by the Commonwealth Fund and the Robert Wood Johnson Foundation through the Center for the Analysis of Health Practices, Harvard School of Public Health.

\section{References}

1 English P C. Failure to thrive without organic reason. Pediatr Ann 1978; 7: 774-81.

2 Shaheen E, Alexander D, Truskowsky M, Barbero G. Failure to thrive: a retrospective profile. Clin Pediatr (Phila) 1968; 7: 255-61.

3 Coleman R W, Provence S. Environmental retardation (hospitalism) in infants living in families. Pediatrics 1957; 19: 285-92.

4 Glaser K, Eisenberg L. Maternal deprivation. Pediatrics $1956 ; 18: 626-42$.

5 Hannaway P J. Failure to thrive. A study of 100 infants and children. Clin Pediatr (Phila) 1970; 9: 96-9.
6 Bullard D M, Jr, Dexter M, Glaser H H, Hegarty M C, Pivchik E C. Failure to thrive in the neglected child. Am J Orthopsychiatry 1967; 37: 680-90.

7 Ambuel J P, Harris B. Failure to thrive. Ohio State Med J 1963; 59: 997-1001.

8 Oates R K, Yu J S. Children with non-organic failure to thrive. A community problem. Med J Aust 1971; ii: 199-203.

9 Sills $\mathbf{R}$ H. Failure to thrive: the role of clinical and laboratory evaluation. Am J Dis Child 1978; 132: 967-9.

10 Pollitt E. Failure to thrive: socioeconomic, dietary intake, and mother-child interaction data. Fed Proc 1975; 34: 1593-7.

Correspondence to Dr Donald M Berwick, Center for the Analysis of Health Practices, Harvard School of Public Health, 677 Huntington Avenue, Boston, Massachusetts 02115, USA.

Received 16 November 1981 